\title{
Effect of VSR invariant Chern-Simon Lagrangian on photon polarization
}

\author{
Alekha C. Nayak * Ravindra K. Verma † $^{\dagger}$ and Pankaj Jain ${ }^{\ddagger}$ \\ Department of Physics, Indian institute of Technology, Kanpur \\ Kanpur, India- 208016
}

\begin{abstract}
We propose a generalization of the Chern-Simon (CS) Lagrangian which is invariant under the $\operatorname{SIM}(2)$ transformations but not under the full Lorentz group. We study the effect of such a term on radiation propagating over cosmological distances. We find that the dominant effect of this term is to produce circular polarization as radiation propagates through space. We use the circular polarization data from distant radio sources in order to impose a limit on this term.
\end{abstract}

\section{INTRODUCTION}

Symmetry is the guiding principle of modern physics. The Standard Model (SM), which is based on Lorentz invariance, provides a successful description of nature and has passed all experimental tests. However, it is believed to be the low energy limit of an ultimate theory at the Planck scale energy. At this scale, unification of quantum field theory with gravity leads to possible violation of Lorentz symmetry [1]. However it is difficult to probe Lorentz violation at LHC because the signal is supressed by ratio of electroweak scale $\left(M_{W}\right)$ to Planck mass $\left(M_{P l}\right)$, i.e. $M_{W} / M_{P l} \approx 10^{-17}[2]$. In a supersymmetric (SUSY) theory the signal may be suppressed more strongly as $\left(M_{S U S Y} / M_{P l}\right)^{2}$, where $M_{S U S Y}$ is the scale of SUSY breaking [3, 4].

Several terrestial [5], astrometric [6, 7] and astrophysical tests have been conducted on Lorentz violation which impose stringent limits on its violation. Different theoretical models have been proposed on possible depatures from Lorentz invariance [1, 3, 4, 8 15]. Colladay and Kostelecky [2] consider the Standard Model Extention (SME) in which Lorentz symmetry is spontaneously violated. Carroll et al. [16] consider a CS term in 3+1 dimension, which is gauge invariant but breaks Lorentz invariance. The authors introduce an external four vector which breaks Lorentz invariance. This term is local and rotates the plane of polarization of photon due to different velocities of left- and right-circularly polarized photon.

We consider $\operatorname{SIM}(2)$ invariant CS term which shows partial gauge invariance but breaks Lorentz invariance. $\operatorname{SIM}(2)$ is the proper subgroup of Lorentz group as developed by Cohen and Glashow [17] and termed Very Special Relativity (VSR). Adjoining one of the discrete symmetry such as $\mathrm{P}, \mathrm{T}, \mathrm{CP}$ or CT with Lorentz subgroup enlarges it to the full Lorentz group. In this paper, we introduce nonlocal operator $\frac{n_{\alpha}}{n \cdot \partial}$ in the CS term in $3+1$ dimension. This operator violates Lorentz invariance while

\footnotetext{
* acnayak@iitk.ac.in

$\dagger$ ravindkv@iitk.ac.in

$\ddagger$ pkjain@iitk.ac.in
}

respecting $\operatorname{SIM}(2)$ invariance. As we shall see, the modified nonlocal CS term splits the photon into two different polarization states which travel with different velocities. This implies violation of parity and Lorentz invariance in the theory. We find that the dominant effect of this term is to generate circular polarization as the electromagnetic wave travels through space. Furthermore the effect is dominant at low frequencies. Using the circular polarization data of radio sources from the MOJAVE (Monitoring of Jets in active galactic nuclei (AGN) with Very Long Baseline Array (VLBA) Experiments) program [18], we impose a limit on the Lorentz violating parameter.

This paper is organised as follows: in Sec. (III) we present the VSR invariant CS Lagrangian and derive the resulting photon dispersion relation. We then obtain the formulas for the Stokes parameters in this model. In Sec. (III) we extract the Lorentz invariance violating parameter by the standard $\chi^{2}$ minimization procedure. Finally, we conclude in Sec. (IV).

\section{THEORY}

The well established Maxwell's theory of electrodynamics is based on gauge and Lorentz invariance. The Lagrangian density for massless photons is given by

$$
\mathcal{L}=-\frac{1}{4} F_{\alpha \beta} F^{\alpha \beta}
$$

where $F_{\alpha \beta}=\partial_{\alpha} A_{\beta}-\partial_{\beta} A_{\alpha}$ is electromagnetic tensor. Eq. (1) is invariant under the gauge transformation $A_{\alpha} \rightarrow A_{\alpha}+\partial_{\alpha} \varphi$. A photon mass term breaks the gauge invariance of the Lagrangian and experimental data imposes a stringent limit on this term. We propose a $\operatorname{SIM}(2)$ invariant nonlocal CS term which can be written as,

$$
\mathcal{L}_{c s}=\frac{\Gamma}{2} \frac{n_{\alpha}}{n \cdot \partial} A_{\beta} \tilde{F}^{\alpha \beta}
$$

Here $\tilde{F}^{\alpha \beta}$ is dual electromagnetic tensor, $\Gamma$ is a parameter of dimension mass square and $n^{\alpha}=(1,0,0,1)$. This is manifestly not Lorentz invariant [19] but is invariant under $\operatorname{SIM}(2)$ transformations. The corresponding generators are $T_{1}=K_{x}+J_{y}, T_{2}=K_{y}-J_{x}$, rotations $\left(J_{z}\right)$ and boosts $\left(K_{z}\right)$ about z-axis. Here $\mathbf{J}$ and $\mathbf{K}$ are the generators of rotations and boosts respectively. Under 
a boost along z-axis $\left(K_{z}\right)$, the preferred vector $n$ transforms as $n^{\alpha} \rightarrow e^{\phi} n^{\alpha}$. However $\frac{n_{\alpha}}{n \cdot \partial}$ is homogeneous in $n$, and hence is $\operatorname{SIM}(2)$ invariant.

Under the gauge transformation $A_{\alpha} \rightarrow A_{\alpha}+\partial_{\alpha} \varphi$, the variation of $\operatorname{SIM}(2)$ modified $\mathrm{CS}$ term is

$$
\begin{aligned}
& \Delta \mathcal{L}^{\prime}{ }_{c s}=\frac{\Gamma}{2}\left(\frac{n_{\alpha}}{n \cdot \partial}\right) \partial_{\beta} \varphi \tilde{F}^{\alpha \beta} \\
& =\frac{\Gamma}{2} \partial_{\beta}\left(\frac{n_{\alpha}}{n \cdot \partial} \varphi \tilde{F}^{\alpha \beta}\right)
\end{aligned}
$$

where we have used the fact that $n^{\alpha}$ is a constant vector and $\partial_{\beta} \tilde{F}^{\alpha \beta}=0$. The remaining term is a surface term and would normally give null contribution to the action. However in the present case, this term also involves the inverse of $n \cdot \partial$. We find that this term vanishes in all cases except if the derivative $\partial_{\alpha}$ is taken in the direction of $n^{\alpha}$. In this case the derivative operation cancels with the inverse operator $1 / n \cdot \partial$ and we obtain a finite contribution. In order to eliminate this violation of gauge invariance we impose the constraint,

$$
n \cdot A=0
$$

This constraint is not invariant under Lorentz transformations but is invariant under $\operatorname{SIM}(2)$ transformations [20]. Hence we can consistently impose it within our framework. We also point out that the vector field $A_{\mu}$ does not form an irreducible representation of $\operatorname{SIM}(2)$ and hence it is not necessary for us to work with the full vector field. In fact the vector potential can be split into four independent one dimensional $\operatorname{SIM}(2)$ fields $[20]$.

The complete Lagrangian density in presence of conserved current $J^{\alpha}$ is

$$
\mathcal{L}_{T}=-\frac{1}{4} F_{\alpha \beta} F^{\alpha \beta}-J^{\alpha} A_{\alpha}+\frac{\Gamma}{2} \frac{n_{\alpha}}{n \cdot \partial} A_{\beta} \tilde{F}^{\alpha \beta}
$$

The equation of motion for the Lagrangian density $\mathcal{L}_{T}$ is

$$
\partial_{\beta} F^{\beta \alpha}=J^{\alpha}-\Gamma \frac{n_{\lambda}}{n \cdot \partial} \tilde{F}^{\lambda \alpha}
$$

The modified Maxwell's equations from Eq. (6) are given by

$$
\begin{aligned}
& \nabla \cdot \mathbf{E}=\rho+\Gamma \frac{1}{n \cdot \partial} \mathbf{n} \cdot \mathbf{B} \\
& -\partial_{t} \mathbf{E}+\nabla \times \mathbf{B}=\mathbf{J}+\Gamma \frac{1}{n \cdot \partial}(\mathbf{B}-\mathbf{n} \times \mathbf{E})
\end{aligned}
$$

Here $\mathbf{n}$ is a unit vector along the $z$ axis. The two homogeneous Maxwell's equations are

$$
\begin{aligned}
& \nabla \cdot \mathbf{B}=0 \\
& \nabla \times \mathbf{E}=-\frac{\partial \mathbf{B}}{\partial t}
\end{aligned}
$$

Using Eqs. (8b) and (77), the source free wave equation takes the form

$\nabla(\nabla \cdot \mathbf{E})-\nabla^{2} \mathbf{E}+\frac{\partial^{2} \mathbf{B}}{\partial t^{2}}=\Gamma \frac{\partial}{\partial t}\left(\frac{1}{n \cdot \partial} \mathbf{B}\right)-\Gamma \frac{\partial}{\partial t}\left(\frac{1}{n \cdot \partial} \mathbf{n} \times \mathbf{E}\right)$
The operator $\frac{1}{n \cdot \partial}$ becomes,

$$
\frac{1}{n \cdot \partial}=\frac{1}{\partial_{t}+\partial_{z}}=\int d t_{+}
$$

where $t_{+}=\frac{t+z}{2}$. Eq. (9) further simplifies to

$$
\left(\omega^{2}-k^{2}\right) \mathbf{E}+(\mathbf{k} \cdot \mathbf{E}) \mathbf{k}=\frac{\Gamma}{\omega-k \cos \theta}(\mathbf{k} \times \mathbf{E}-\omega \mathbf{n} \times \mathbf{E})
$$

In the present case, for the source free modified Maxwell's equation $\mathbf{k} \cdot \mathbf{E} \neq 0$, so the longitudinal component of the photon polarization (proportional to $\Gamma$ ) is not zero. It acquires a small value compared to the transverse components. Since the vector $\mathbf{n}$ is along the $\mathrm{z}$-direction and the wave propagation vector, $\hat{\mathbf{k}}$, makes an angle $\theta$ with $\mathbf{n}$, the electric field can be expressed as,

$$
\mathbf{E}=E_{x} \hat{\mathbf{x}}+E_{y z} \hat{\mathbf{p}}+E_{k} \hat{\mathbf{k}}
$$

where

$$
\begin{aligned}
& \hat{\mathbf{p}}=-\hat{\mathbf{y}} \cos \theta+\hat{\mathbf{z}} \sin \theta \\
& \hat{\mathbf{k}}=\hat{\mathbf{z}} \cos \theta+\hat{\mathbf{y}} \sin \theta \\
& \hat{\mathbf{n}}=\hat{\mathbf{z}}
\end{aligned}
$$

Comparing the $\mathrm{x}-, \mathrm{y}-$ and $\mathrm{z}-$ component of Eq. (11), we get

$$
\begin{aligned}
& \left(\omega^{2}-k^{2}\right) E_{x}= \\
& \frac{\Gamma}{(\omega-k \cos \theta)}\left\{(k-\omega \cos \theta) E_{y z}+\omega \sin \theta E_{k}\right\} \\
& \Gamma E_{x}=\left(\omega^{2}-k^{2}\right) \cos \theta E_{y z}-\omega^{2} \sin \theta E_{k} \\
& \frac{\Gamma k \sin \theta}{\omega-k \cos \theta} E_{x}=-\left(\omega^{2}-k^{2}\right) \sin \theta E_{y z}-\omega^{2} \cos \theta E_{k}
\end{aligned}
$$

Using Eq. (14), we get the following dispersion relation

$$
\omega^{2}-k^{2}= \pm i \Gamma
$$

Here the + and - signs correspond to right- and lefthanded polarized photons. Hence we find that the modified SIM(2) invariant CS term leads to different dispersion relations for the right and left handed polarizations.

From Eq. (14), the relation between the components of electric fields for the two solutions in Eq. 15] are given by

$$
\begin{aligned}
& E_{x} \approx \mp i \frac{(\omega-k \cos \theta)}{(k-\omega \cos \theta)} E_{y z} \\
& E_{k} \approx \frac{\left(\omega^{2}-k^{2}\right) \sin \theta}{\omega^{2}(1-\cos \theta)} E_{y z}
\end{aligned}
$$

where we have kept only the leading order terms in $\Gamma$. Using Eq. (15), we find that the two eigenmodes of propagation are,

$$
\left|E_{+}\right\rangle \approx\left(\begin{array}{c}
\frac{(\omega-k \cos \theta)}{(k-\omega \cos \theta)} \\
i \\
\frac{i\left(\omega^{2}-k^{2}\right) \sin \theta}{\omega^{2}(1-\cos \theta)}
\end{array}\right),\left|E_{-}\right\rangle \approx\left(\begin{array}{c}
\frac{(\omega-k \cos \theta)}{(k-\omega \cos \theta)} \\
-i \\
\frac{-i\left(\omega^{2}-k^{2}\right) \sin \theta}{\omega^{2}(1-\cos \theta)}
\end{array}\right)
$$


In the limit $\omega \approx k$ the corresponding eigenvalues are given by

$$
k \approx \omega \mp \frac{i \Gamma}{2 \omega} \equiv k_{ \pm}
$$

where we have used Eq. (15).

In the limit, $\Gamma \rightarrow 0$, the two vectors in Eq. 17 correspond to the left and right circular polarizations, i.e.

$$
\left|E_{+}\right\rangle=\left(\begin{array}{c}
1 \\
i \\
0
\end{array}\right),\left|E_{-}\right\rangle=\left(\begin{array}{c}
1 \\
-i \\
0
\end{array}\right)
$$

Eq. (18) implies that two polarization modes travel with different velocity which is an indication of parity violation. This model leads to a significant contribution to circular polarization at low frequencies, which we will discuss in the next section.

Let us label the axes along $\hat{\mathbf{x}}, \hat{\mathbf{p}}$ and $\hat{\mathbf{k}}$ by $x_{1}, x_{2}$ and $x_{3}$ respectively. Hence our wave is propagating along the $x_{3}$ direction and the two transverse directions are taken to be along $x_{1}$ and $x_{2}$. An electric state vector, at any given time, can be written as a linear combination of two state vectors given in Eq. (17).i.e

$$
\begin{aligned}
\left|E\left(x_{3}, t\right)\right\rangle & =\frac{E_{+}(0)}{\sqrt{2}}\left(\begin{array}{c}
\frac{\left(\omega-k_{+} \cos \theta\right)}{\left(k_{+}-\omega \cos \theta\right)} \\
i \\
\frac{i\left(\omega^{2}-k_{+}^{2}\right) \sin \theta}{\omega^{2}(1-\cos \theta)}
\end{array}\right) e^{i\left(k_{+} x_{3}-w t\right)} \\
& +\frac{E_{-}(0)}{\sqrt{2}}\left(\begin{array}{c}
\frac{\left(\omega-k_{-} \cos \theta\right)}{\left(k_{-}-\omega \cos \theta\right)} \\
-i \\
\frac{-i\left(\omega^{2}-k_{-}^{2}\right) \sin \theta}{\omega^{2}(1-\cos \theta)}
\end{array}\right) e^{i\left(k_{-} x_{3}-w t\right)}
\end{aligned}
$$

Here $E_{3}\left(x_{3}, t\right)$ component of the state vector $\left|E\left(x_{3}, t\right)\right\rangle$ is very small and we are interested in determining the change in the photon polarization in the plane perpendicular to the photon propagation. Using Eq. (20), we obtain,

$$
\begin{aligned}
& E_{1}\left(x_{3}, t\right)=\frac{1}{\sqrt{2}}\left(P_{+} E_{+}(0) e^{i k_{+} x_{3}}\right. \\
&\left.+P_{-} E_{-}(0) e^{i k_{-} x_{3}}\right) e^{-i \omega t} \\
& E_{2}\left(x_{3}, t\right)=\frac{1}{\sqrt{2}}\left(i E_{+}(0) e^{i k_{+} x_{3}}\right. \\
&\left.\quad-i E_{-}(0) e^{i k_{-} x_{3}}\right) e^{-i \omega t}
\end{aligned}
$$

where

$$
P_{ \pm}=\frac{\omega-k_{ \pm} \cos \theta}{k_{ \pm}-\omega \cos \theta}
$$

The electric vector rotates in the plane perpendicular to the direction of propagation, when the two photon polarization modes travel with different velocity. Hence, the polarization state of photon changes after propagation over a large distance. It can be determined by calculating the Stokes parameter I,Q,U,V.
We assume that the wave is unpolarized at source and calculate its polarization after propagation through a distance $x_{3}$. The Jones matrix for unpolarized electromagnetic wave is

$$
J=\left(\begin{array}{ll}
1 & 0 \\
0 & 1
\end{array}\right)
$$

Using this as the initial condition, we calculate the Stokes parameters after the photon has travelled a distance $x_{3}$. These are given by,

$$
\begin{aligned}
I & =2 \cosh \left(\frac{\Gamma x_{3}}{\omega}\right) \\
Q & =0 \\
U & =\frac{2 \Gamma \cot ^{2}\left(\frac{\theta}{2}\right) \sinh ^{2}\left(\frac{\Gamma x_{3}}{2 \omega}\right)}{\omega^{2}} \\
V & =2 \sinh \left(\frac{\Gamma x_{3}}{\omega}\right)
\end{aligned}
$$

Keeping only the leading order in $\Gamma$, we obtain,

$$
I=2, Q=0, U=0, V=\frac{2 \Gamma}{\omega} x_{3}
$$

This implies that an initially unpolarized wave acquires circular polarization upon propagation. The polarization state does not depend upon the direction of propagation of photon with respect to the VSR preferred axis. From Eq. (25), we obtain,

$$
\xi \equiv \frac{V}{I}=\frac{\Gamma}{\omega} x_{3}
$$

So far we have confined our analysis to a flat spacetime. However we need to compute the change in polarization in an expanding Universe since we are interested in sources located at redshifts comparable to unity. We consider a spatially flat Universe. The propagation of electromagnetic wave follows the same equations as given above but with time replaced by conformal time and the distance $x_{3}$ replaced by comoving distance [21, 22]. Besides this the overall energy density in the wave decreases due to expansion. However this effect is not relevant for calculation of shift in polarization. Consider a source at a redshift $z$. Its comoving distance $x_{3}$ is given by [23]

$$
x_{3}=\frac{1}{a_{0} H_{0}} \int_{\frac{1}{1+z}}^{1} \frac{\mathrm{d} x}{x^{2} \sqrt{\Omega_{\Lambda}+\Omega_{M} x^{-3}}}
$$

where $H_{0}$ is Hubble constant, $a_{0}$ is scale parameter at present epoch, $\Omega_{\Lambda}$ is the ratio of vacuum energy density to critical density and $\Omega_{M}$ is the ratio of non-relativistic matter density to critical density. Hence Eq(26) becomes

$$
\begin{array}{r}
\xi \equiv \frac{V}{I}=\frac{\Gamma}{\omega a_{0} H_{0}} \int_{\frac{1}{1+z}}^{1} \frac{\mathrm{d} x}{x^{2} \sqrt{\Omega_{\Lambda}+\Omega_{M} x^{-3}}} \\
=\beta \int_{\frac{1}{1+z}}^{1} \frac{\mathrm{d} x}{x^{2} \sqrt{\Omega_{\Lambda}+\Omega_{M} x^{-3}}}
\end{array}
$$


where $\beta=\frac{\Gamma}{\omega a_{0} H_{0}}$ is dimensionless. We find that the circular polarization depends on the redshift of the source. Furthermore the polarization generated increases with decrease in frequency. Hence the effect is dominant at low frequencies, such as, radio waves. In comparison the dominant effect in the case of the local Lorentz violating term, studied in Ref. [16] is to produce a frequency independent rotation of linear polarization. We point out that mixing of hypothetical pseudoscalars of very low mass with photons in a background magnetic field also generates circular polarization 24 28]. In this case the effect is found to increase with frequency and is limited by the stringent constraints that have been imposed on the circular polarization which may be generated at optical frequencies [29].

In more generality, the theoretical model can be expressed as,

$$
\xi=\beta \int_{\frac{1}{1+z}}^{1} \frac{\mathrm{d} x}{x^{2} \sqrt{\Omega_{\Lambda}+\Omega_{M} x^{-3}}}+\xi_{0}
$$

where we have added a constant $\xi_{0}$ to the model in order to allow for the possibility that the mean value of circular polarization over the sample is not zero. In the limit $\beta=0, \xi_{0}$ is the mean value of circular polarization over the entire sample. For $\beta \neq 0$ we determine both the parameters, $\beta$ and $\xi_{0}$, by making a $\chi^{2}$ fit to data.

\section{RESULTS}

In this section, we determine the best fit parameters by using the $\chi^{2}$ minimisation procedure for the circularly polarized light emitted from radio jets associated with AGN. Here we use the circular polarization data from the MOJAVE program, which contains 133 bright, mostly compact radio-loud AGN in the northern sky. With VLBA facilities, circular polarization of the AGN jet sample at $15 \mathrm{Ghz}$ with flux density 1.5 Jy has been observed. We only consider sources with redshift $z$ greater than 0.25 for which local effects are absent. We find that after this cut only 102 sources remain in the data set.

We extract the Lorentz violating parameter $(\beta)$ by minimizing $\chi^{2}$ for the model given in Eq. 29. The resulting $\chi^{2}$ is compared with the null model $\xi=\xi_{0}$ in order to determine the significance of the fit. For the null model we find that the mean value $\xi_{0}=0.38$ with $\chi^{2}=347.6$. The Lorentz violating model, Eq. 29, yields $\beta=0.067 \pm 0.12, \xi_{0}=0.40$ with minimum value of $\chi^{2}=347.1$. Hence we do not find a significant signal of Lorentz violation in the data. The corresponding best fit is shown in Fig. (1). Using the extracted value of $\beta$ we find that the one sigma limit on the Lorentz violating parameter $\Gamma$ is $\left(13 \times 10^{-29} \mathrm{GeV}\right)^{2}$.

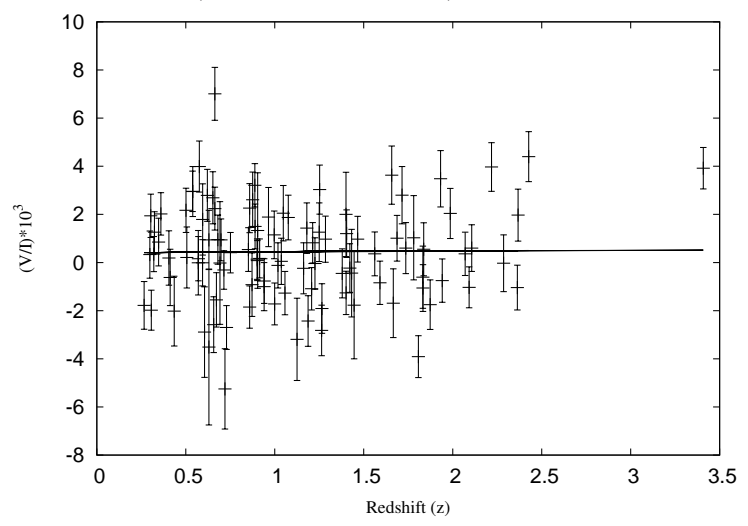

FIG. 1. The fit to the circular polarization data from distant radio galaxies.

\section{CONCLUSION}

We have proposed a modified Chern-Simon term which breaks the full Lorentz invariance but is invariant under $\operatorname{SIM}(2)$ transformations. The term is non-local and depends on a prefered vector, $n^{\alpha}$. The term also respects gauge invariance provided we impose the condition, $n \cdot A=0$, on the gauge potential, $A_{\alpha}$. This condition is invariant under the $\operatorname{SIM}(2)$ transformation and can be imposed consistently in this theory. The non-local CS term changes the dispersion relation of photon. Hence it changes the polarization of the electromagnetic waves travelling over large distances. We find that at leading order, an initially unpolarized picks up circular polarization upon propagation. We test the predicted signal by using the circular polarization data from distant radio galaxies. We do not find a significant signal of violation of Lorentz invariance and impose a stringent limit on the Lorentz violation parameter.
[1] J. Collins, A. Perez, D. Sudarsky, L. Urrutia, and H. Vucetich, Phys. Rev. Lett. 93, 191301 (2004), grqc/0403053.

[2] D. Colladay and V. A. Kostelecky, Phys. Rev. D58, 116002 (1998), hep-ph/9809521.

[3] S. Groot Nibbelink and M. Pospelov, Phys. Rev. Lett. 94, 081601 (2005), hep-ph/0404271.
[4] P. Jain and J. P. Ralston, Phys. Lett. B621, 213 (2005), hep-ph/0502106.

[5] R. J. Kennedy and E. M. Thorndike, Phys. Rev. 42, 400 (1932).

[6] Z. Bay and J. White, Acta Physica Academiae Scientiarum Hungaricae 51, 273 (1981).

[7] J. Mller and M. Soffel, Phys. Lett. A 198, 71 (1995), ISSN 0375-9601. 
[8] V. A. Kostelecky and M. Mewes, Phys. Rev. D80, 015020 (2009), 0905.0031.

[9] R. Bluhm and V. A. Kostelecky, Phys. Rev. D71, 065008 (2005), hep-th/0412320.

[10] M. Berger and V. A. Kostelecky, Phys. Rev. D65, 091701 (2002), hep-th/0112243.

[11] S. M. Carroll, J. A. Harvey, V. A. Kostelecky, C. D. Lane, and T. Okamoto, Phys. Rev. Lett. 87, 141601 (2001), hep-th/0105082.

[12] D. Colladay and V. A. Kostelecky, Phys. Lett. B511, 209 (2001), hep-ph/0104300.

[13] D. Colladay and V. A. Kostelecky, Phys. Rev. D55, 6760 (1997), hep-ph/9703464.

[14] A. V. Kostelecky and N. Russell, Phys. Lett. B693, 443 (2010), 1008.5062.

[15] S. R. Coleman and S. L. Glashow, Phys. Rev. D59, 116008 (1999), hep-ph/9812418.

[16] S. M. Carroll, G. B. Field, and R. Jackiw, Phys. Rev. D41, 1231 (1990).
[17] A. G. Cohen and S. L. Glashow, Phys. Rev. Lett. 97, 021601 (2006), hep-ph/0601236.

[18] D. C. Homan and M. Lister, Astron. J. 131, 1262 (2006), astro-ph/0511838.

[19] A. G. Cohen and S. L. Glashow (2006), hep-ph/0605036.

[20] J. Vohánka, Phys. Rev. D 85, 105009 (2012), 1112.1797.

[21] S. M. Carroll and G. B. Field, Phys. Rev. D 43, 3789 (1991).

[22] P. Tiwari, Phys. Rev. D 86, 115025 (2012), 1207.0606.

[23] S. Weinberg, Cosmology (Oxford University Press, 2008).

[24] P. Sikivie, Physical Review Letters 51, 1415 (1983).

[25] L. Maiani, R. Petronzio, and E. Zavattini, Physics Letters B 175, 359 (1986).

[26] G. Raffelt and L. Stodolsky, Phys. Rev. D 37, 1237 (1988).

[27] P. Jain, S. Panda, and S. Sarala, Phys. Rev. D 66, 085007 (2002), hep-ph/0206046.

[28] S. Das, P. Jain, J. P. Ralston, and R. Saha, JCAP 6, 002 (2005), hep-ph/0408198.

[29] A. Payez, J. R. Cudell, and D. Hutsemékers, JCAP 7, 041 (2012), 1204.6187. 